\title{
McGill
}

\section{Nonapeptide influences on social behaviour: effects of vasotocin and isotocin on shoaling and interaction in zebrafish}

\author{
Charlotte M. Lindeyer, Esther M. A. Langen, William T. \\ Swaney, and Simon M. Reader \\ Behaviour, Volume 152, Issue 7-8, pages $897-915$
}

(2015)

doi: $10.1163 / 1568539 X-00003261$

http://booksandjournals.brillonline.com/content/journals/10.1163/1568539x $-00003261$ 


\section{Nonapeptide influences on social behaviour: effects of vasotocin and isotocin on shoaling and interaction in zebrafish}

Charlotte M. Lindeyer, Esther M. A. Langen, William T. Swaney, and Simon M. Reader 


\section{Introduction}

Animals engage in a wide range of social behaviours which vary enormously across taxa and species. In contrast to the phenotypic variation in social behaviour, there appears to be extensive regulatory overlap between species, with the nonapeptides oxytocin and vasopressin repeatedly demonstrated to be important regulators of multiple mammalian social behaviours including parental care (Pedersen, 2013), pair bonding (Winslow et al., 1993), affiliative behaviour (Madden \& Clutton-Brock, 2011), social recognition (Bielsky et al., 2004), aggression (Albers et al., 2006) and even human social interactions (Meyer-Lindenberg et al., 2011). Furthermore, differences in nonapeptide release or receptor distribution have been strongly implicated in interspecies variation in social behaviour (Insel \& Shapiro, 1992; Bester-Meredith et al., 1999), as well as intra-species population differences (Beiderbeck et al., 2007) and individual differences in social behaviour (Francis et al., 2000). However, sociality is far from a uniquely mammalian attribute and accumulating evidence implicates the nonapeptides in the regulation of social behaviour in other taxa (Moore et al., 2005; Godwin \& Thompson, 2012).

The influence of nonapeptides on putatively complex forms of sociality has been extensively researched, with a particular focus in recent years on nonapeptide effects on affiliative and prosocial behaviours, often ignoring one of the most fundamental forms of sociality, association with conspecifics or grouping behaviour (Goodson \& Kingsbury, 2011). A notable exception to this is the extensive work of Goodson and colleagues characterising the role that nonapeptides play in grouping and sociality in estrildid finch species. For example, they have shown that variation in nonapeptide neuron number and nonapeptide receptor density is associated with between-species variation in grouping behaviour (Goodson \& Wang, 2006), and that pharmacological manipulations targeting nonapeptide receptors modulate individual grouping propensities (Goodson et al., 2009). However the influence of nonapeptides on grouping behaviour in other taxa is relatively understudied, prompting us to investigate the regulatory roles of nonapeptides on grouping behaviour in fish. Fish are the largest vertebrate class, exhibit an extensive and varied array of social behaviours (Brown et al., 2006) and express the homologous nonapeptides vasotocin (AVT) and isotocin (IT), permitting nonapeptides 
to be investigated in a socially rich taxon that is evolutionarily distant from mammals and birds. Fish also offer excellent opportunities for exploring grouping behaviour as many species form cohesive groups, and grouping propensities can be readily quantified.

Although much more work has been done in mammals, evidence indicates that nonapeptides influence multiple social behaviours in fish, including dominance interactions, aggression, parental behaviour, social communication and courtship (Goodson \& Bass, 2000; Lema \& Nevitt, 2004; Greenwood et al., 2008; O’Connell et al., 2012). Fewer studies have addressed grouping behaviour and related phenomena. Butterflyfish species (family Chaetodontidae) with greater territorial aggression and smaller social group sizes have larger preoptic AVT neurons and denser telencephalic AVT fibres than non-territorial, shoaling species (Dewan et al., 2008, 2011). In goldfish ( Carassius auratus), time in proximity to conspecifics ('social approach') is modulated by nonapeptide administration: IT reduces it while AVT increases it (Thompson \& Walton, 2004). These effects are seen in both sexes, however they appear to be dependent on baseline levels of social approach (Thompson \& Walton, 2004) and on reproductive state in this seasonally-breeding species (Walton et al., 2010).

We wished to determine whether IT and AVT influence grouping behaviour in fish and so investigated how nonapeptides affect this fundamental component of social behaviour in zebrafish ( Danio rerio). The zebrafish, a small freshwater fish native to South Asia (Spence et al., 2008), is a model system for genetics and developmental biology and is increasingly being used to study behaviour. Zebrafish readily shoal and nonapeptides have previously been implicated in the regulation of zebrafish social behaviours. Neuronal localization of AVT within the preoptic area is restricted to large magnocellular neurons in dominant zebrafish and to small parvocellular neurons in subordinates (Larson et al., 2006). AVT levels have also been shown to vary according to dominance status, although whether AVT expression is higher in dominant individuals (Filby et al., 2010) or in subordinates (Pavlidis et al., 2011) appears to depend on the precise makeup of the social group and the duration of such group housing. Administration of AVT has been shown to reduce aggression in zebrafish (Filby et al., 2010), while both AVT and IT have been shown to increase preferences for a samestrain shoal in zebrafish (Braida et al., 2012). We administered nonapeptides and 
putative nonapeptide receptor antagonists to individual zebrafish and measured shoaling and social interaction in a social behaviour test with a novel stimulus shoal.

Based on Thompson \& Walton's (2004) findings in goldfish, we predicted that IT would increase and AVT would decrease shoaling and social interaction. In the goldfish, a seasonal breeder, these responses are dependent on reproductive state (Walton et al., 2010). However, we did not take reproductive state into account here as reproduction in the zebrafish, also a cyprinid, is driven by food availability and so they breed year round in captivity (Spence et al., 2008).

\section{Material and methods}

\subsection{Subjects and housing}

A total of 125 adult female zebrafish (4-5 months old) were used as subjects (mean mass $\pm \mathrm{SE}=0.33 \pm 0.004 \mathrm{~g}$ ). Twenty additional adult females (mean mass $\pm \mathrm{SE}$ $=0.35 \pm 0.004 \mathrm{~g}$ ), unfamiliar to the subjects and housed separately, served as stimulus shoals in the behavioural tests. We used females to minimise aggression and dominance effects on shoaling behaviour. All subjects were bred in-house at our departmental aquarium and were experimentally naïve $\mathrm{F} 2$ descendants of fish purchased from a commercial supplier ('wild type' strain, Ruijsbroek, Maassluis, The Netherlands). Subjects were housed in a large tank $(150 \times 50 \mathrm{~cm})$, stimulus shoal fish in a small tank $(80 \times 50 \mathrm{~cm})$. Once subjects had been tested, they were rehoused in separate small tanks $(80 \times 50 \mathrm{~cm})$ by treatment group. Due to this rehousing, a further 20 adult females were included in the home tank so that the final subjects to be tested were not socially isolated. All tanks were maintained at $26 \pm 1^{\circ} \mathrm{C}$ with $30 \mathrm{~cm}$ of water and were enriched with artificial plants, pot shelters and gravel. Lights were on a $12 \mathrm{~h} / 12 \mathrm{~h}$ schedule with lights on at $08: 00 \mathrm{~h}$ and no natural light. Fish were fed twice daily (at 09:00 and 17:00 h) with 'TetraMin' flake food (Tetra, Melle, Germany) in the morning and bloodworm (Chironomidae) or Daphniaspp. in the afternoon. On test days, fish were given a single combined feeding after the conclusion of testing. Water quality $(\mathrm{pH}$, nitrates and nitrites) was checked weekly and tanks were cleaned fortnightly. 


\subsection{Administration treatment groups}

Subjects were selected at random from their home tank and assigned to one of five treatment groups immediately prior to testing: (1) IT (AbD Serotec, Kidlington, UK), (2) AVT (Bachem, Bubendorf, Switzerland), (3) a putative IT receptor antagonist (IT-a), (4) a putative AVT receptor antagonist (AVT-a), or (5) 0.9\% saline. The IT-a was the selective oxytocin receptor antagonist desGly-NH 2,d(CH 2) 5[ d-Tyr 2,Thr 4]OVT (Manning et al., 1995) and the AVT-a was the selective vasopressin 1a receptor antagonist d(CH 2) 5[Tyr(Me) 2,Dab 5]AVP (Chan et al., 1996), both generous gifts of Professor M. Manning of the University of Toledo, Toledo, OH, USA.

Each group consisted of 25 subjects. To address any possible observer bias, treatment order was pseudo-randomly determined using Microsoft Excel's RAND function, and a second researcher prepared and labelled the solutions to be administered so that the researcher conducting tests was blind to which group was being tested on each day. Tests were conducted over two weeks and to minimise order effects half of the subjects from each treatment group were tested in the first week and the remainder in the second week. Time of day of testing was counterbalanced across groups to minimise intergroup variance due to circadian effects.

\subsection{Treatment dosages and administration}

All substances were dissolved in $0.9 \%$ saline and administered at a dose of 10 $\mu \mathrm{g} / \mathrm{g}$ body weight. Doses were based on peripheral administration studies in zebrafish and other small fish (Carneiro et al., 2003; Lema \& Nevitt, 2004; Filby et al., 2010). For administration, subjects were caught in the home tank with a net, weighed in water and then placed on a wet tissue for intraperitoneal injection with a $10 \mu \mathrm{l}$ Hamilton syringe and $30 \mathrm{G}$ needle, with injection volumes no more than $6 \mu \mathrm{l}$. The administration procedure took approximately $20 \mathrm{~s}$, after which subjects were placed in the social behaviour test tank.

\subsection{Grouping test}

We measured effects of administrations on zebrafish shoaling and social interaction. A large tank $(150 \times 50 \mathrm{~cm})$ was divided into three areas by transparent plastic partitions: two side compartments each $11.5 \mathrm{~cm}$ wide and a central $127 \mathrm{~cm}$ wide 
compartment (Figure 1). Prior to testing, eight stimulus shoal fish were caught with a net and placed into a transparent plastic container $(11.5 \times 12.5 \mathrm{~cm})$ filled with $26 \pm 1^{\circ} \mathrm{C}$ water which was then placed in one of the side compartments while the opposite compartment remained empty. The stimulus shoal fish were chosen at random from the pool of 20 fish and used for 2-3 consecutive trials. Shoal location was randomised after every two trials. The central subject compartment was divided into three zones by boundaries drawn on the front of the tank: a central 'neutral' zone and outer 'shoaling' and 'no-shoal' zones $10 \mathrm{~cm}$ or 3-4 body lengths from each plastic partition, following Pitcher's (1983) definition of shoaling. Directly after administration, the subject was placed in a $7 \mathrm{~cm}$ diameter transparent plastic cylinder in the middle of the central compartment. After $5 \mathrm{~min}$ for recovery, acclimatisation and to enable administered substances to reach the brain, the cylinder was smoothly pulled upwards by rope and pulley to release the subject and start the 10-min trial. The post-injection recovery period was the same across treatments so that recovery from the injection procedure did not differentially influence the different treatment groups. We used a short recovery time due to the short plasma half-life of nonapeptides (Gozdowska et al., 2013). Subject behaviour was scored live with JWatcher V1.o ( http://www.jwatcher.ucla.edu) and recorded with a Megapixel Pro webcam (Trust International, Dordrecht, The Netherlands) and AMCap 9.20 software. After testing, subjects were moved to their post-testing housing tank. All stimulus shoal fish were weighed after being used in tests. Subjects were weighed prior to administration and again one week later to check for possible effects of administration on weight and health.

\subsection{Statistical analyses}

We measured shoaling behaviour and interaction with the shoal. Subjects were defined as shoaling when they were within the shoaling zone, and as interacting when they were swimming head first against the transparent partition, in a manner directed towards the stimulus shoal. This behaviour was readily distinguished from general shoaling behaviour when fish swam within the shoaling zone but did not directly approach the partition. Persistent swimming directed at the shoal may indicate greater motivation to socially interact than does mere presence in the shoaling zone, and so we used the shoaling and interaction measures to differentiate between grouping and more 
active social interest. A similar interaction measure has recently been demonstrated to give different results from grouping measures (Kelly et al., 2011), and thus could reflect a different aspect of social behaviour and motivation.

The dependent variables were total time in the shoaling zone, total time in the no-shoal zone, total time spent interacting, latency to shoal, latency to interact and time interacting as a proportion of time shoaling. We also analysed the number of transitions across zone boundaries as a combined measure of activity and stress. Treatment group was a fixed effect, shoal position, subject mass and mean mass of the stimulus shoal were covariates. We used generalised linear models (GLMs) to investigate the effect of treatment on the behavioural measures. Time and latency data were right skewed and so were analysed with a gamma family of errors. To control for overdispersion, proportional data (interaction as a proportion of shoaling) were analysed with a quasibinomial family of errors and count data (transitions across zones) were analysed with a quasi-Poisson family of errors (Crawley, 2007). Treatment contrasts were employed to assess the effects of each administration relative to saline, with an alpha significance level of 0.05. To explore differences between treatments we defined three planned comparisons of interest (AVT vs. AVT-a, IT vs. IT-a, AVT vs. IT) and ran additional GLMs with a pre-defined a priori contrast matrix (package Epi) and a Bonferroni adjusted critical alpha level ( $\alpha=0.0167$ ) for multiple comparisons. These comparisons were chosen to compare effects of each nonapeptide with their putative receptor antagonist and the two nonapeptides with each other. All statistical tests were two tailed and data are expressed as means $\pm \mathrm{SE}$. Body mass of subjects, mean mass of stimulus shoals and shoal position were not found to be significant predictors of shoaling behaviour $(\mathrm{p} \geqslant 0.1)$ and therefore are not reported below. Analyses were performed in R Project 2.10.1.

\subsection{Ethical note}

The experiment was approved by our local Animal Experimentation Committee ('Dier Experimenten Commissie') under licence 2010.I.12.263, and conformed to Dutch animal welfare legislation and to the ASAB/ABS Guidelines for the Use of Animals in Research. Our Animal Experimentation Committee and university veterinarians discussed and observed our proposed procedure before experiments began. We strived 
to minimise distress by making the administration procedure as short as possible and not using anaesthetic: although this would have led to some discomfort during the actual injection, the far shorter duration of the whole procedure was judged to reduce overall distress. No adverse effects of any of the tested substances were observed on behaviour or health, and as noted below, there were no significant differences between groups in either individual weight or weight gain after administration. Fish were killed at the conclusion of experiments by immersion in ice water for 1 min (following Blessing et al., 2010), as this is the fastest, most effective and most humane method of killing small tropical fish such as the zebrafish (Wilson et al., 2009).

\section{$\underline{\text { 3. Results }}$}

\subsection{Interaction with the shoal}

Interaction behaviour was only observed in the shoaling zone and never at the partition in the no-shoal zone, suggesting it was directed specifically at the stimulus shoal and was an effective measure of social behaviour. Both AVT and AVT-a significantly reduced interaction time compared to saline (GLM: AVT, t $24=2 \cdot 34, \mathrm{p}=$ $0.02 ;$ AVT-a, t $24=2.03, p=0.04$, Figure 2B). Other administrations did not significantly differ from saline (GLM: $t 24 \leqslant 1.60, p>0.1$ ). IT-treated fish spent less time interacting than IT-a treated fish, but not significantly so (GLM with a priori contrasts: $\mathrm{z} 24=1.94, \mathrm{p}=0$. 05 ). AVT also significantly increased the latency to start interacting with the stimulus shoal compared to saline and IT (GLM: AVT vs. saline, t 24 $=2.88, \mathrm{p}=0.005 ;$ GLM with a priori contrasts: AVT vs. IT, z 24 = 2.42, p = 0 . 015 , Figure $3 \mathrm{~B})$.

To further investigate interaction behaviour, we analysed time spent interacting as a proportion of total time spent shoaling (Figure $2 \mathrm{C}$ ). AVT, AVT-a and IT administrations significantly decreased the proportion of shoaling time spent interacting compared to saline (GLM: AVT, t $24=5 \cdot 55, \mathrm{p}<0$. o01; AVT-a, t $24=2$. $46, \mathrm{p}=0.02 ; \mathrm{IT}, \mathrm{t} 24=2.76, \mathrm{p}=0.01)$. Additionally, the proportion of shoaling time spent interacting was significantly lower after AVT administration than AVT-a or IT (GLM with a priori contrasts: AVT vs. AVT-a, z $24=3.49, \mathrm{p}=0$. 001 ; AVT vs. IT, z 
$24=3.21, p=0.001)$. IT significantly decreased the proportion of shoaling time spent interacting compared to IT-a (GLM with a priori contrasts: $z 24=3.09, p=0$. 002 ).

\subsection{Shoaling}

Time in the shoaling zone differed significantly between treatment groups (Figure 2A). AVT-a significantly reduced time shoaling compared to saline (GLM: t $24=$ $2.58, \mathrm{p}=0.01$ ), however other groups did not significantly differ from the saline group (GLM: $t 24 \leqslant 1.51, p>0.1$ ). Shoaling was significantly higher after AVT administration than IT or AVT-a administration (GLM with a priori contrasts: AVT vs. IT, z $24=2.48, p=0$. 01; AVT vs. AVT-a, z 24 = 3. 76, p < o . 001$)$.

Subjects demonstrated a strong tendency to associate with the stimulus shoal, spending more time in the shoaling zone (mean $\pm \mathrm{SE}=184.4 \pm 27.9 \mathrm{~s}$ ) than the no-shoal zone (mean $\pm \mathrm{SE}=46.9 \pm 14.4 \mathrm{~s}$ ), however there were effects of treatment: subjects in all groups spent significantly more time in the shoaling zone than the no-shoal zone, except for the AVT-a group (Wilcoxon paired signed-ranks tests: AVT-a, $\mathrm{U}=\mathbf{2 2 5}, \mathrm{N}=$ $25, \mathrm{p}=0.09$; other groups, $\mathrm{U} \geqslant 264, \mathrm{~N}=25$ per group, $\mathrm{p}<0$. 005 in all cases). AVT and AVT-a both significantly increased time in the no-shoal zone compared to saline (GLM: t 24 $=2.02, p=0.05 ;$ t $24=2.03, p=0.04$, respectively). The planned comparisons did not reveal significant differences between peptide treatments in time spent in the no-shoal zone (GLM with a priori contrasts: $z 24 \leqslant 1.34, p \geqslant 0.2$ ).

Subjects typically swam away from the cylinder and back and forth in the neutral zone immediately after release, before swimming to either end of the tank. There were no statistically significant effects on latency to begin shoaling (Figure 3A). AVT-treated fish were slower to begin shoaling than both saline and IT-treated fish, but not significantly so (GLM: AVT vs. saline, t $24=2.58, p=0$. 09 ; GLM with a priori contrasts: AVT vs. IT, z $24=1.78, \mathrm{p}=0.08$ ).

\subsection{Other measures}

AVT, AVT-a and IT significantly increased the frequency of transitions across zones compared to saline (GLM: AVT, t $24=3.42, \mathrm{p}=0$. o01; AVT-a, t $24=2.29, \mathrm{p}$ $=0.02 ;$ IT, t $24=1.98, p=0.05$, Figure 4 ). The planned comparisons revealed no 
significant differences in zone transitions between AVT vs. IT, AVT vs. AVT-a or IT vs. IT-a (GLM with a priori contrasts: $\mathrm{z} 24 \leqslant 1.90, \mathrm{p}>0$. 05 ). Subjects' body mass (mean $\pm \mathrm{SE}=0.39 \pm 0.05 \mathrm{~g}$ ) did not differ significantly between administration treatments either before (Linear Model (LM): t $24 \leqslant 1.64, \mathrm{p}>0$. 1 ) or after testing (LM: $\mathrm{t} 24 \leqslant 0$. $98, \mathrm{p}>0$. 3 ). Subjects gained weight in the week after testing (Wilcoxon signed rank test: $\mathrm{W}=5734 \cdot 5, \mathrm{~N}=150, \mathrm{p}<0.0001$ ), but weight gain did not differ significantly between treatments (LM, t $24 \leqslant 1.07, \mathrm{p}>0.3$ ).

\section{Discussion}

Our results suggest that nonapeptides, and AVT in particular, modulate grouping behaviour in zebrafish, as administrations of both AVT and an AVT receptor antagonist had clear effects on subjects' social interaction and shoaling behaviour with a stimulus shoal. AVT-treated subjects were slower to interact and spent less time interacting with the shoal, both in absolute terms and as a proportion of time spent shoaling, than any other treatment. While AVT did not affect shoaling time, shoaling was reduced by AVT-a compared to both saline and AVT. In contrast to these diverging effects on shoaling time, the effects of AVT-a on absolute interaction time were similar to those of AVT. These differences in the responses to AVT and AVT-a across shoaling and interaction were unanticipated, and suggest differences in how AVT regulates the interaction and grouping behaviours we measured. While these social behaviours were modulated by AVT manipulations, we found little evidence of a role for IT in the regulation of social grouping in zebrafish, with no detectable responses to IT-a, and the only significant effect of IT administration being a reduction in interaction time as a proportion of time shoaling.

\subsection{Vasotocin}

We saw a marked reduction in social interaction in fish that received AVT, mirroring findings in goldfish in which AVT inhibited social approach (Thompson \& Walton, 2004; Thompson et al., 2008). This effect of AVT on social interaction also has parallels in the findings of Filby et al. (2010) who showed that AVT reduced aggressive behaviours, including chasing of conspecifics, in small groups of zebrafish. The observed 
reduction in social interaction in response to AVT may be linked to this previously reported effect of AVT on aggressive interactions: diminishing close interaction may decrease the chance of aggression, or diminished aggression may motivate less close approach of conspecifics. Intriguingly, we found that despite its effects on social interaction, AVT did not modify shoaling behaviour, as AVT-treated fish spent at least as much time as control subjects in proximity to the stimulus shoal, suggesting that these two social behaviours are differentially sensitive to AVT and that they may be decoupled.

These differing effects of AVT and AVT-a administration suggest that there is a behavioural distinction between shoaling tendency and social interaction in zebrafish. There are similarities between these findings and recent data on grouping in the gregarious zebra finch (Kelly et al., 2011). In this study, a vasopressin 1a receptor antagonist decreased preferences for larger group sizes but increased social contact time, findings that, like ours, indicate regulatory separation between grouping behaviour and social interaction. These findings emphasise the importance of distinguishing between general tendencies to associate with conspecifics and more focused social interaction when studying social behaviour and grouping. Moreover, they demonstrate that nonapeptides do not act as blanket up- or down-regulators of even apparently similar social behaviours, cautioning against extrapolation of the influence of nonapeptides across social behaviour more generally.

Contrary to our expectations, AVT and AVT-a did not have opposing effects across our different measures of social behaviour. In some cases the effects were in different directions (total time shoaling), or the size of the effect was significantly different (proportion of time interacting with the shoal), however on other measures the responses were similar (total interaction time). These different results may be due to unanticipated effects of using a putative AVT antagonist in zebrafish, which although a highly specific antagonist of the mammalian vasopressin 1a receptor (Manning et al., 2008), has not been pharmacologically characterised in fish. Differences between mammalian and zebrafish AVT receptor binding sites may impact the affinity and efficacy of the AVT-a we used. Furthermore, nonapeptide receptors in teleosts and mammals are also not uniformly equivalent: zebrafish have been shown to have two receptors homologous to the mammalian vasopressin 1a receptor, both of which are expressed in the brain (Iwasaki et al., 2013). Concurrent administration of AVT and 
AVT-a would assist in determining whether AVT-a functions as a true antagonist in zebrafish and so would block effects of exogenous AVT mediated via AVT receptors. It is also possible that behaviour may have been influenced by peripheral, physiological responses to intraperitoneal injection rather than through direct central effects.

However, it should be noted that behavioural responses to different neuropeptides have been shown to be similar in fish, whether administered centrally or peripherally (Olson et al., 1978). In mice, peripherally administered nonapeptides elicit behavioural responses via central effects (Ring et al., 2006), indicating that they cross the bloodbrain barrier. Peripheral administration has been also used to study many diverse social behaviours in fish (Carneiro et al., 2003; Lema \& Nevitt, 2004; Santangelo \& Bass, 2006; O'Connell et al., 2012; Reddon et al., 2012) and the varied and complex effects reported suggest that the behavioural responses are not simply due to physiological perturbation.

While we found that AVT reduced social interaction, Braida et al. (2012) recently reported that AVT administration in zebrafish increased preferences for same-strain shoals in a dose-dependent fashion. The two studies address different questions: we measured the influence of nonapeptides on shoaling tendencies, while Braida and colleagues examined specifically the effects of nonapeptides on strain preferences in choice tests, rather than on social approach directly. Moreover, Braida et al. (2012) used doses of AVT and IT based on studies of intracerebroventricular administration of oxytocin and vasopressin in mice, orders of magnitude lower than the doses we and other researchers have typically employed for intraperitoneal administration studies in fish (Carneiro et al., 2003; Lema \& Nevitt, 2004; Filby et al., 2010). The inverted-U dose-response effect of AVT on same-strain shoaling preferences reported by Braida et al. (2012) might predict an increase in shoaling at the dose utilised in our study, however we did not see such an effect.

\subsection{Isotocin}

IT significantly decreased the proportion of shoaling time spent interacting compared to saline, but did not have any effect on actual time spent shoaling or interacting. These findings should not be over-interpreted but suggest IT influences grouping behaviour to a lesser degree than AVT. While IT has been shown to stimulate 
social approach in goldfish, effects are only seen in subjects with lower baseline levels of social approach (Thompson \& Walton, 2004). Since zebrafish show very strong shoaling tendencies (Buske \& Gerlai, 2011) our ability to detect any influence of IT administration may have been limited by a ceiling effect. Another possibility is that IT does not increase gregarious behaviour in zebrafish, as supported by recent findings that certain doses of IT decreased preferences for same-strain zebrafish (Braida et al., 2012).

The focus of much nonapeptide research on prosocial behaviour has resulted in a widespread narrative that nonapeptides, and oxytocin in particular, are the primary regulators of prosocial behaviour (Barraza \& Zak, 2013). However our data and those of others suggest that across vertebrates this view is overly simplistic, with extensive variation in the role and importance of nonapeptides across species, behaviours and contexts. For example, while IT increases submissive behaviour in a cooperativelybreeding cichlid (Reddon et al., 2012), it has no effect on aggressive interactions in the beaugregory damselfish Stegastes leucostictus(Santangelo \& Bass, 2006), and blockade of nonapeptide signalling disrupts neither novel nor established pair bonding in a monogamous cichlid (Oldfield \& Hofmann, 2011). In our experiments, IT had no significant effects on grouping behaviour, suggesting at the very least that this homologue of oxytocin does not function as a broadly prosocial neuropeptide in zebrafish and does not mediate increased group cohesion. Furthermore, while AVT and IT have sometimes been described as 'male' and 'female' nonapeptides, the responses of the female subjects to AVT but not to IT, as well as the data of others (Walton et al., 2010) do not support this view.

\subsection{Activity and stress responses}

Nonapeptides have been shown to be involved in neuroendocrine responses to stress in teleosts, in particular AVT which stimulates cortisol release via ACTH (Balment et al., 2006). We thus considered whether the reductions in shoaling and interaction after nonapeptide administration might be a consequence of changes in stress responses. However no specifically stress-related behaviours such as freezing or dashing (Egan et al., 2009) were observed, nor did we see any effects of treatment on health or growth post-testing. The increased switching rates seen in the AVT, AVT-a and IT administered subjects compared to the control group could indicate increased stress or 
activity in these fish, as putative anxiolytics have been shown to reduce swimming activity (Levin et al., 2007). However, external stressors typically cause decreases in zone switching and swimming in zebrafish (Bass \& Gerlai, 2008; Cachat et al., 2010), suggesting that elevated activity may not always be an indicator of stress. Recent work in a cichlid has shown that vasotocin administration increases circulating cortisol but decreases swimming activity (Huffman et al., 2014). As zebrafish show tighter shoaling in response to stress (Speedie \& Gerlai, 2008), the decrease in social interaction we observed in response to AVT, AVT-a and IT treatment suggests that these administrations were not simply increasing stress responses but were modulating sociality. However, additional research will be necessary to tease apart direct nonapeptide effects on social behaviour from indirect effects mediated by HPI axis activation.

\subsection{Conclusions}

Our results demonstrate that AVT manipulations affect shoaling and social interaction, although our study also suggests that these are discrete behaviours that are differentially regulated by AVT and its receptors. Our findings offer further evidence that nonapeptides have a broad role in regulating social behaviour across vertebrates (Goodson, 2008) but also provide evidence that nonapeptides influence perhaps the most fundamental aspect of sociality, the tendency to associate with conspecifics in a group. Further comparisons of relatively simple social behaviours across species will increase our understanding of the neural underpinnings of social behaviour and its evolution (O'Connell \& Hofmann, 2011), and of the degree to which nonapeptide regulation of sociality has been conserved throughout vertebrate evolution.

Acknowledgements Go to section...

We thank Prof. M. Manning of the University of Toledo for kindly providing the isotocin and vasotocin antagonists. We thank Willem van der Wal and Paul Westers (Julius Center at UMC Utrecht), Rahia Mashoodh and Han de Vries for statistical advice, Adam Reddon for discussion, and Henk Schriek, Ko van Rootselaar and Henk Westland for their assistance with animal care. We gratefully acknowledge funding from the Utrecht University High Potentials programme. 


\section{$\underline{\text { References }}$}

Albers H.E. Dean A. Karom M.C. Smith D. Huhman K.L. ( 2006). Role of V1a vasopressin receptors in the control of aggression in Syrian hamsters. - Brain Res. 1073-1074: 425- 430.

Balment R.J. Lu W. Weybourne E. Warne J.M. ( 2006). Arginine vasotocin a key hormone in fish physiology and behaviour: a review with insights from mammalian models. - Gen. Comp. Endocrinol. 147: 9- 16.

Barraza J.A. Zak P.J. ( 2013). Oxytocin instantiates empathy and produces prosocial behaviors. - In: Oxytocin, vasopressin and related peptides in the regulation of behavior( Choleris E. Pfaff D.W. Kavaliers M. , eds). Cambridge University Press, Cambridge, p. 331- 342.

Bass S.L.S. Gerlai R. ( 2008). Zebrafish ( Danio rerio) responds differentially to stimulus fish: the effects of sympatric and allopatric predators and harmless fish. - Behav. Brain Res. 186: 107- 117.

Beiderbeck D.I. Neumann I.D. Veenema A.H. ( 2007). Differences in intermale aggression are accompanied by opposite vasopressin release patterns within the septum in rats bred for low and high anxiety. - Eur. J. Neurosci. 26: 3597- 3605.

Bester-Meredith J.K. Young L.J. Marler C.A. (1999). Species differences in paternal behavior and aggression in Peromyscusand their associations with vasopressin immunoreactivity and receptors. - Horm. Behav. 36: 25-38.

Bielsky I.F. Hu S.B. Szegda K.L. Westphal H. Young L.J. ( 2004). Profound impairment in social recognition and reduction in anxiety-like behavior in vasopressin V1a receptor knockout mice. - Neuropsychopharmacology 29: 483- 493.

Blessing J.J. Marshall J.C. Balcombe S.R. ( 2010). Humane killing of fishes for scientific research: a comparison of two methods. - J. Fish Biol. 76: 2571- 2577.

Braida D. Donzelli A. Martucci R. Capurro V. Busnelli M. Chini B. Sala M. ( 2012). Neurohypophyseal hormones manipulation modulate social and anxiety-related behavior in zebrafish. - Psychopharmacology 220: 319- 330.

Brown C. Laland K.N. Krause J. , eds ( 2006). Fish cognition and behavior. - Blackwell, Oxford. 
Buske C. Gerlai R. ( 2011). Shoaling develops with age in zebrafish ( Danio rerio). Prog. Neuropsychopharmacol. Biol. Psychiatr. 35: 1409- 1415.

Cachat J.M. Canavello P.R. Elegante M.F. Bartels B.K. Elkhayat S.I. Hart P.C. Tien A.K. Tien D.H. Beeson E. Mohnot S. Laffoon A.L. Stewart A.M. Gaikwad S. Wong K. Haymore W. Kalueff A.V. ( 2010). Modeling stress and anxiety in zebrafish. - In: Zebrafish models in neurobehavioral research( Kalueff A.V. Cachat J.M. , eds). Humana Press, Totowa, NJ, p. 73-88.

Carneiro L.A. Oliveira R.F. Canário A.V.M. Grober M.S. ( 2003). The effect of arginine vasotocin on courtship behaviour in a blenniid fish with alternative reproductive tactics. - Fish Physiol. Biochem. 28: 241- 243.

Chan W.Y. Wo N.C. Cheng L.L. Manning M. ( 1996). Isosteric substitution of Asn 5in antagonists of oxytocin and vasopressin leads to highly selective and potent oxytocin and V1a receptor antagonists: new approaches for the design of potential tocolytics for preterm labor. - J. Pharmacol. Exp. Ther. 277: 999- 1003. Crawley M.J. ( 2007). The R book. - Wiley, Chichester.

Dewan A.K. Maruska K.P. Tricas T.C. ( 2008). Arginine vasotocin neuronal phenotypes among congeneric territorial and shoaling reef butterflyfishes: species, sex and reproductive season comparisons. - J. Neuroendocrinol. 20: 1382- 1394.

Dewan A.K. Ramey M.L. Tricas T.C. ( 2011). Arginine vasotocin neuronal phenotypes, telencephalic fiber varicosities, and social behavior in butterflyfishes (Chaetodontidae): potential similarities to birds and mammals. - Horm. Behav. 59: 56- 66.

Egan R.J. Bergner C.L. Hart P.C. Cachat J.M. Canavello P.R. Elegante M.F. Elkhayat S.I. Bartels B.K. Tien A.K. Tien D.H. Mohnot S. Beeson E. Glasgow E. Amri H. Zukowska Z. Kalueff A.V. ( 2009). Understanding behavioral and physiological phenotypes of stress and anxiety in zebrafish. - Behav. Brain Res. 205: 38- 44.

Filby A.L. Paull G.C. Hickmore T.F. Tyler C.R. ( 2010). Unravelling the neurophysiological basis of aggression in a fish model. - BMC Genomics 11: 498.

Francis D.D. Champagne F.C. Meaney M.J. ( 2000). Variations in maternal behaviour are associated with differences in oxytocin receptor levels in the rat. $-\mathrm{J}$. Neuroendocrinol. 12: 1145-1148. 
Godwin J. Thompson R. ( 2012). Nonapeptides and social behavior in fishes. - Horm. Behav. 61: 230- 238.

Goodson J.L. ( 2008). Nonapeptides and the evolutionary patterning of sociality. Prog. Brain Res. 170: 3- 15 .

Goodson J.L. Bass A.H. ( 2000). Forebrain peptides modulate sexually polymorphic vocal circuitry. - Nature 403: 769- 772.

Goodson J.L. Kingsbury M.A. ( 2011). Nonapeptides and the evolution of social group sizes in birds. - Front. Neuroanat. 5: 13.

Goodson J.L. Wang Y. ( 2006). Valence-sensitive neurons exhibit divergent functional profiles in gregarious and asocial species. - Proc. Natl. Acad. Sci. USA 103: 17013- 17017 .

Goodson J.L. Schrock S.E. Klatt J.D. Kabelik D. Kingsbury M.A. ( 2009). Mesotocin and nonapeptide receptors promote estrildid flocking behavior. - Science 325: 862866.

Gozdowska M. Ślebioda M. Kulczykowska E. ( 2013). Neuropeptides isotocin and arginine vasotocin in urophysis of three fish species. - Fish Physiol. Biochem. 39: 863-869.

Greenwood A.K. Wark A.R. Fernald R.D. Hofmann H.A. ( 2008). Expression of arginine vasotocin in distinct preoptic regions is associated with dominant and subordinate behaviour in an African cichlid fish. - Proc. Roy. Soc. Lond. B: Biol. Sci. 275: 2393- 2402.

Huffman L.S. Hinz F.I. Wojcik S. Aubin-Horth N. Hofmann H.A. ( 2014). Arginine vasotocin regulates social ascent in the African cichlid fish Astatotilapia burtoni . - Gen. Comp. Endocrinol., in press , DOI: 10.1016/j.ygcen.2014.03.004.

Insel T.R. Shapiro L.E. ( 1992). Oxytocin receptor distribution reflects social organization in monogamous and polygamous voles. - Proc. Natl. Acad. Sci. USA 89: 5981- 5985 .

Iwasaki K. Taguchi M. Bonkowsky J.L. Kuwada J.Y. ( 2013). Expression of arginine vasotocin receptors in the developing zebrafish CNS. - Gene Expr. Patterns 13: 335- 342.

Kelly A.M. Kingsbury M.A. Hoffbuhr K. Schrock S.E. Waxman B. Kabelik D. Thompson R.R. Goodson J.L. ( 2011). Vasotocin neurons and septal V1a-like receptors 
potently modulate songbird flocking and responses to novelty. - Horm. Behav. 60: 12- 21.

Larson E.T. O’Malley D.M. Melloni J.R.H. ( 2006). Aggression and vasotocin are associated with dominant-subordinate relationships in zebrafish. - Behav. Brain Res. 167: 94- 102.

Lema S.C. Nevitt G.A. ( 2004). Exogenous vasotocin alters aggression during agonistic exchanges in male Amargosa River pupfish ( Cyprinodon nevadensis amargosae). - Horm. Behav. 46: 628- 637.

Levin E.D. Bencan Z. Cerutti D.T. ( 2007). Anxiolytic effects of nicotine in zebrafish. Physiol. Behav. 90: 54- 58.

Madden J.R. Clutton-Brock T.H. ( 2011). Experimental peripheral administration of oxytocin elevates a suite of cooperative behaviours in a wild social mammal. Proc. Roy. Soc. Lond. B: Biol. Sci. 278: 1189- 1194.

Manning M. Miteva K. Pancheva S. Stoev S. Wo N.C. Chan W.Y. (1995). Design and synthesis of highly selective in vitroand in vivouterine receptor antagonists of oxytocin: comparisons with Atosiban. - Int. J. Pept. Protein Res. 46: 244- 252.

Manning M. Stoev S. Chini B. Durroux T. Mouillac B. Guillon G. ( 2008). Peptide and non-peptide agonists and antagonists for the vasopressin and oxytocin V1a, V1b, V2 and OT receptors: research tools and potential therapeutic agents. - Prog. Brain Res. 170: 473- 512.

Meyer-Lindenberg A. Domes G. Kirsch P. Heinrichs M. ( 2011). Oxytocin and vasopressin in the human brain: social neuropeptides for translational medicine. - Nat. Rev. Neurosci. 12: 524- 538.

Moore F.L. Boyd S.K. Kelley D.B. ( 2005). Historical perspective: hormonal regulation of behaviors in amphibians. - Horm. Behav. 48: 373- 383.

O'Connell L.A. Hofmann H.A. ( 2011). Genes, hormones, and circuits: an integrative approach to study the evolution of social behavior. - Front. Neuroendocrinol. 32: 320- 335 .

O’Connell L.A. Matthews B.J. Hofmann H.A. ( 2012). Isotocin regulates paternal care in a monogamous cichlid fish. - Horm. Behav. 61: 725- 733.

Oldfield R.G. Hofmann H.A. ( 2011). Neuropeptide regulation of social behavior in a monogamous cichlid fish. - Physiol. Behav. 102: 296- 303. 
Olson R.D. Kastin A.J. Montalbano-Smith D. Olson G.A. Coy D.H. Michell G.F. ( 1978). Neuropeptides and the blood-brain barrier in goldfish. - Pharmacol. Biochem. Behav. 9: 521- 524.

Pavlidis M. Sundvik M. Chen Y.C. Panula P. ( 2011). Adaptive changes in zebrafish brain in dominant-subordinate behavioral context. - Behav. Brain Res. 225: 529- 537.

Pedersen C.A. ( 2013). Oxytocin regulation of maternal behavior: from rodents to humans. - In: Oxytocin, vasopressin and related peptides in the regulation of behavior( Choleris E. Pfaff D.W. Kavaliers M. , eds). Cambridge University Press, Cambridge, p. 148- 182.

Pitcher T.J. ( 1983). Heuristic definitions of fish shoaling behavior. - Anim. Behav. 31: 611- 613 .

Reddon A.R. O'Connor C.M. Marsh-Rollo S.E. Balshine S. ( 2012). Effects of isotocin on social responses in a cooperatively breeding fish. - Anim. Behav. 84: 753- 760.

Ring R. Malberg J. Potestio L. Ping J. Boikess S. Luo B. Schechter L. Rizzo S. Rahman Z. Rosenzweig-Lipson S. ( 2006). Anxiolytic-like activity of oxytocin in male mice: behavioral and autonomic evidence, therapeutic implications. Psychopharmacology 185: 218- 225.

Santangelo N. Bass A.H. ( 2006). New insights into neuropeptide modulation of aggression: field studies of arginine vasotocin in a territorial tropical damselfish. - Proc. Roy. Soc. Lond. B: Biol. Sci. 273: 3085- 3092.

Speedie N. Gerlai R. ( 2008). Alarm substance induced behavioral responses in zebrafish ( Danio rerio). - Behav. Brain Res. 188: 168- 177.

Spence R. Gerlach G. Lawrence C. Smith C. ( 2008). The behaviour and ecology of the zebrafish, Danio rerio . - Biol. Rev. 83: 13- 34.

Thompson R.R. Walton J.C. ( 2004). Peptide effects on social behavior: effects of vasotocin and isotocin on social approach behavior in male goldfish ( Carassius auratus). - Behav. Neurosci. 118: 620- 626.

Thompson R.R. Walton J.C. Bhalla R. George K.C. Beth E.H. ( 2008). A primitive social circuit: vasotocin-substance $\mathrm{P}$ interactions modulate social behavior through a peripheral feedback mechanism in goldfish. - Eur. J. Neurosci. 27: 2285- 2293. 
Walton J.C. Waxman B. Hoffbuhr K. Kennedy M. Beth E. Scangos J. Thompson R.R. ( 2010). Behavioral effects of hindbrain vasotocin in goldfish are seasonally variable but not sexually dimorphic. - Neuropharmacology 58: 126- 134 .

Wilson J.M. Bunte R.M. Carty A.J. ( 2009). Evaluation of rapid cooling and tricaine methanesulfonate (MS222) as methods of euthanasia in zebrafish ( Danio rerio). - J. Am. Ass. Lab. Anim. Sci. 48: 785- 789.

Winslow J.T. Hastings N. Carter C.S. Harbaugh C.R. Insel T.R. ( 1993). A role for central vasopressin in pair bonding in monogamous prairie voles. - Nature 365: 545548. 
Figure 1.

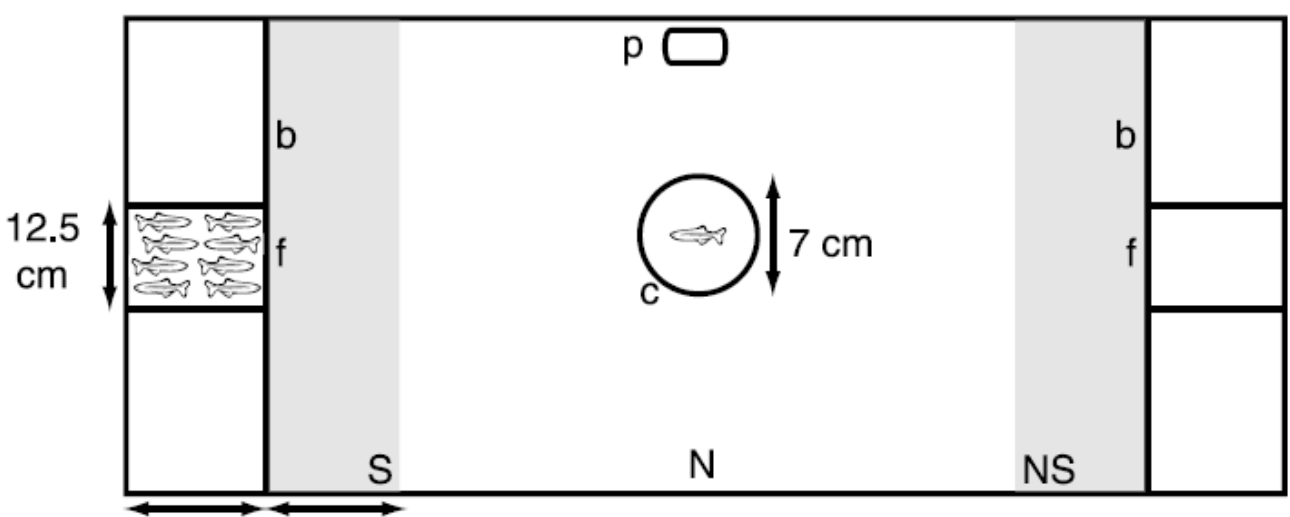

$11.5 \mathrm{~cm} 10 \mathrm{~cm}$

Schematic overview of the experimental apparatus, plan view. Lines were drawn on the outside of a large aquarium $(150 \times 50 \mathrm{~cm})$ marking a neutral zone (N) containing a pump (p), a shoaling zone (S) and a no-shoal zone (NS), the latter two dependant on the location of a stimulus shoal. The subject was released from a transparent cylinder (c) after acclimatisation and its behaviour was recorded for $10 \mathrm{~min}$. A conspecific shoal was placed at random on one side of the tank behind a transparent solid partition $(b)$ in a confined zone $(f)$. Interaction was recorded when subjects were both in the shoaling zone (S) and swimming head first against the partition (b). 
Figure 2.
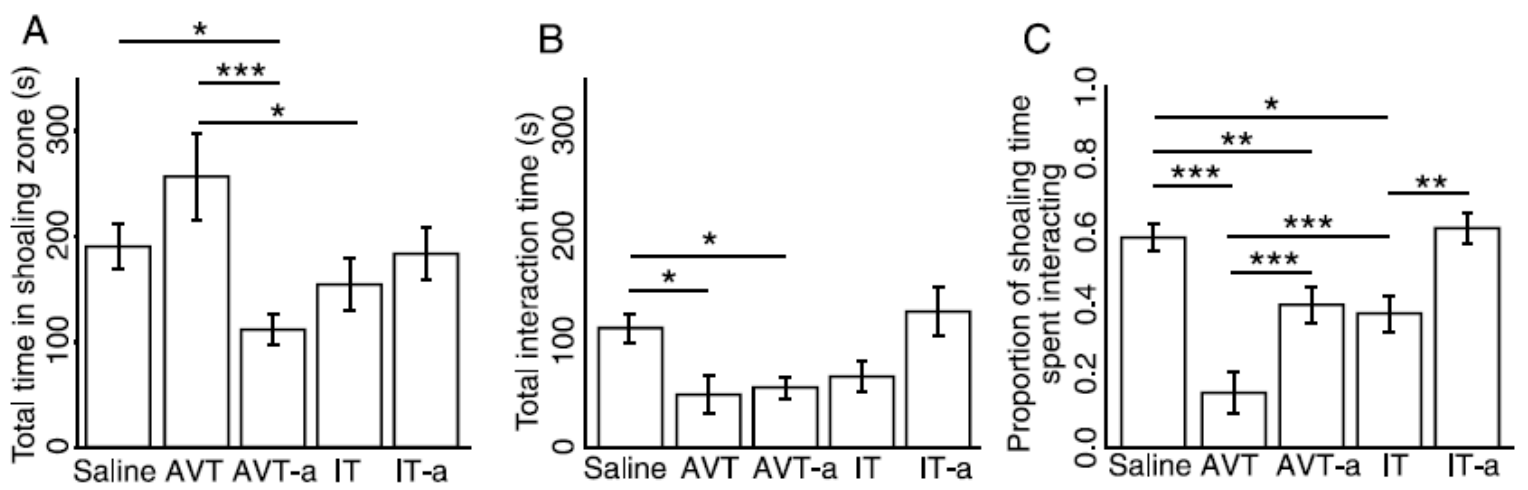

Mean $\pm S E$ values for each treatment for (A) time spent in the shoaling zone, (B) time spent interacting with the shoal and $(C)$ time spent interacting as a proportion of time spent shoaling for each treatment group. * $p \leqslant 0.05, * * p \leqslant 0.01, * * * p \leqslant 0.001$. 
Figure 3.
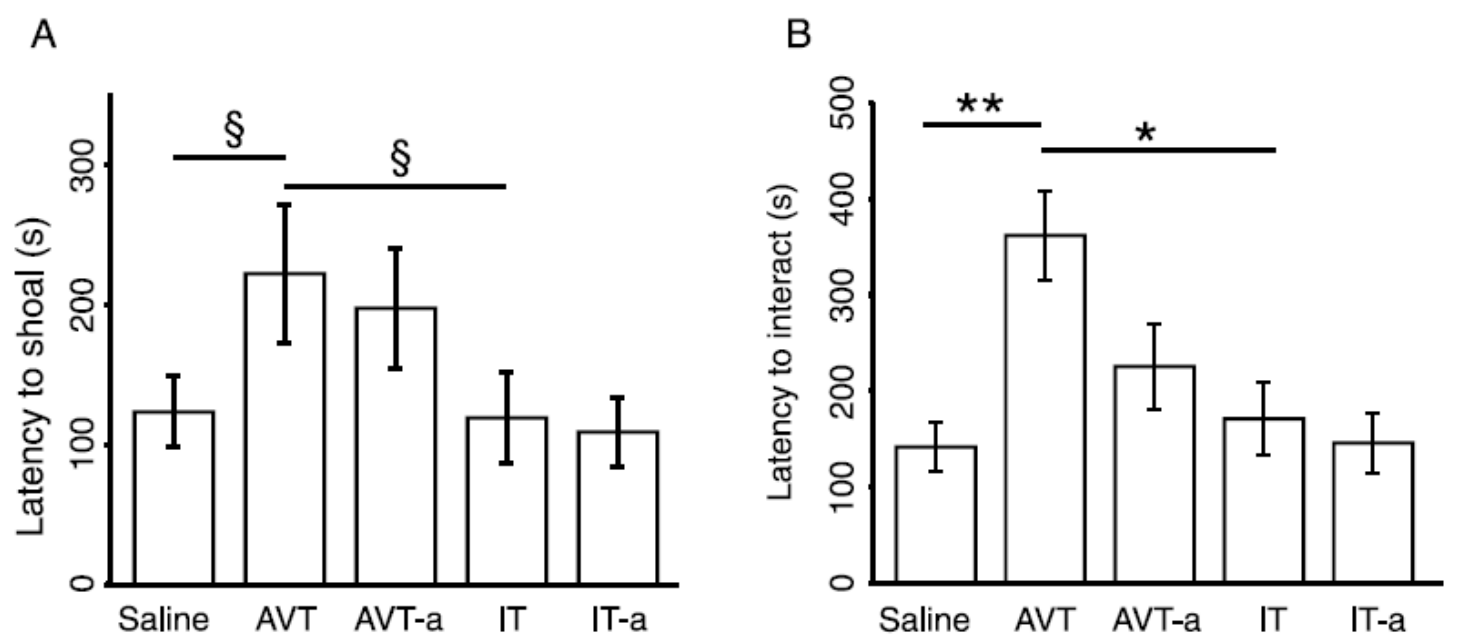

Mean $\pm S E$ values for each treatment for (A) latency to enter the shoaling zone and (B) latency to interact at the partition with the stimulus shoal. $\S p \leqslant 0.1, * p \leqslant 0.05, * *$ $p \leqslant 0.01$. 
Figure 4.

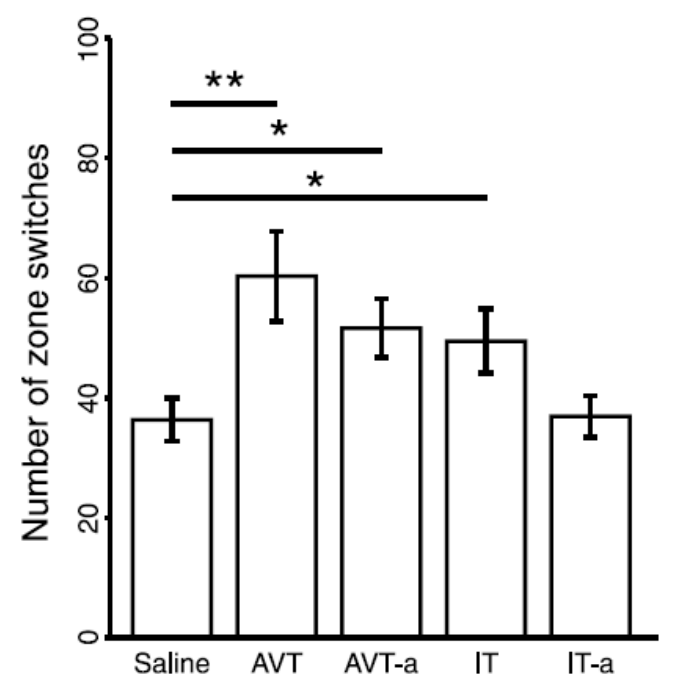

Mean $\pm S E$ number of switches made between the shoaling, neutral and no-shoal zones for each treatment. $* p \leqslant 0.05, * * p \leqslant 0.01, N S: p \geqslant 0.1$. 